\title{
Whole-genome sequencing in health care
}

\section{Recommendations of the European Society of Human Genetics}

\author{
Carla G van $\mathrm{El}^{1}$, Martina C Cornel ${ }^{1,2,3}$, Pascal Borry ${ }^{4}$, Ros J Hastings ${ }^{5}$, Florence Fellmann ${ }^{6}$, Shirley V Hodgson ${ }^{7}$, \\ Heidi C Howard ${ }^{8,9}$, Anne Cambon-Thomsen ${ }^{8,9}$, Bartha M Knoppers ${ }^{10}$, Hanne Meijers-Heijboer ${ }^{11}$, \\ Hans Scheffer ${ }^{12}$, Lisbeth Tranebjaerg ${ }^{13,14,15}$, Wybo Dondorp ${ }^{16,17}$, Guido MWR de Wert ${ }^{3,16,17}$ \\ on behalf of the ESHG Public and Professional Policy Committee
}

European Journal of Human Genetics (2013) 21, 580-584; doi:10.1038/ejhg.2013.46

Keywords: recommendations; whole-genome sequencing; policy

\section{INTRODUCTION}

In recent years, the cost of generating genome information has shown a rapid decline. ${ }^{1,2}$ High-throughput genomic technologies make it possible to sequence the whole exome or genome of a person at a price that is affordable for some health-care systems. More services based on these technologies are now becoming available for patients, raising the issue of how to ensure that these are provided appropriately. In order to determine both the clinical utility of genetic testing and assure a high quality of the analysis, the interpretation and communication of the results must be discussed so that patients can receive appropriate advice and genetic testing. The Public and Professional Policy Committee (PPPC) and the Quality Committee of the European Society of Human Genetics (ESHG) addressed these challenges at a joint workshop in Gothenburg, Sweden, in 2010. ${ }^{3}$ PPPC also organised workshops in Amsterdam, the Netherlands (January 2011 in collaboration with the EU-funded project TECHGENE, January 2012). A report for the Health Council of the Netherlands served as a background document for the PPPC's reflections. ${ }^{4}$ Focusing on the clinical diagnostics setting, this paper is intended to contribute to the discussion and the development of guidelines in this fast-moving field, and provide recommendations for health-care professionals. The paper and recommendations were posted on the ESHG website from 20 June to 1 August 2012 for comment by the membership. The final version was approved by the ESHG Board in December 2012.

\section{CONSIDERATIONS}

The changing landscape of diagnostic genetic testing in health care Until recently, a diagnostic genetic test tended to focus on one specific question. In the case of a clinical suspicion of a monogenic condition,
DNA analysis of one or a few specific genes was performed, whereas in cytogenetics, the whole genome was analysed at a relatively low resolution of 5-10 Mb to answer a defined clinical question. Increasingly, however, diagnostic tests now look at a large panel of genes (eg, genes implied in cardiovascular events) via microarrays, a relatively targeted approach. In addition, non-targeted high-resolution next-generation sequencing techniques may be applied, detecting mutations throughout the genome. Whole-genome- or exome sequencing (WGS, WES) generates an enormous amount of raw data requiring complex bioinformatic analyses to extract useful information. Depending on the aim of the test, the analysis may focus on the entire genome (whole-genome analysis, WGA), the exome (wholeexome analysis), a selection of genes, the high-resolution quantitative comparison between copies of different chromosomes or chromosomal segments, or other selected analyses. Experience with recent changes in DNA-laboratory methods with targeted DNA sequencing or microarrays might be used to improve the understanding of the challenges for professionals, as WGS and WES are introduced into health care. ${ }^{5}$

WES is already in use in several laboratories in a diagnostic setting. ${ }^{6,7}$ As far as WGS is concerned, several groups have sequenced individual genomes, ${ }^{8}$ or are conducting research, such as in the Personal Genome Project. ${ }^{9}$ Proof of principle regarding the clinical utility of WGS followed by WGA has been reported, especially for rare diseases. ${ }^{10-12}$ Moreover, expectations of personalized medicine appear to be more likely through the use of whole-genome technologies, though others point out that it will take much time and effort. ${ }^{13}$ For instance, Ashley et al ${ }^{10}$ provide examples of personalised medicine including pharmacogenomic variants that could guide therapy, and the detection of carrier status of cystic fibrosis that could possibly lead

\footnotetext{
${ }^{1}$ Section Community Genetics, Department of Clinical Genetics and EMGO Institute for Health and Care Research, VU University Medical Center, Amsterdam, The Netherlands; ${ }^{2}$ Center for Medical Systems Biology, Leiden, The Netherlands; ${ }^{3}$ CSG Centre for Society and the Life Sciences, Nijmegen, The Netherlands; ${ }^{4}$ Department of Public Health and Primary Care, KU Leuven, Leuven, Belgium; ${ }^{5}$ CEQA and UK NEQAS for Clinical Cytogenetics, John Radcliffe Hospital, Oxford University Hospitals NHS Trust, Oxford, UK; ${ }^{6}$ Service de Génétique Médicale, CHUV, Lausanne, Switzerland; ${ }^{7}$ Department of Medical Genetics, St George's University of London, London, UK; ${ }^{8}$ Department of Epidemiology and Analyses in Public Health, UMR 1027, Inserm, Toulouse, France; ${ }^{9}$ Faculté de Médecine, Université de Toulouse III. Paul Sabatier, Toulouse, France; 10Department of Human Genetics, Faculty of Medicine, Centre of Genomics and Policy, McGill University, Montreal, Canada; ${ }^{11}$ Section Oncogenetics, Department of Clinical Genetics, VU University Medical Center, Amsterdam, The Netherlands; ${ }^{12}$ Department of Human Genetics, Radboud University Nijmegen Medical Centre, Nijmegen, The Netherlands; ${ }^{3}$ Department of Audiology, H:S Bispebjerg Hospital, Copenhagen, Denmark; 14Wilhelm Johannsen Centre of Functional Genome Research, Department of Cellular and Molecular Medicine (ICMM), Copenhagen, Denmark; ${ }^{15}$ The Panum Institute, University of Copenhagen, Copenhagen, Denmark; ${ }^{16}$ Department of Health, Ethics and Society; Research School CAPHRI, Maastricht University, Maastricht, The Netherlands; ${ }^{17} \mathrm{GROW}$, School for Oncology and Developmental Biology, Maastricht University, Maastricht, The Netherlands *Correspondence: Dr CG van El, Section Community Genetics, Department of Clinical Genetics and EMGO Institute for Health and Care Research, VU University Medical Center, BS7, D450, PO Box 7057, 1007 MB, Amsterdam, The Netherlands, Tel: + 3120444 8867; Fax: 3120444 8181; E-mail: cg.vanel@vumc.nl
} 
to testing of the partner and affect the reproductive options. Furthermore, the detection of the mutations associated with sudden death may lead to cardiologic consultation having management implications. ${ }^{10}$ In cancer research, findings of distinct genetic mutations in the tumours of a patient ${ }^{14}$ may lead to targeted therapeutic strategies. Initial successes in diagnosing hitherto unknown causes and/or predictors of diseases have raised expectations on the wider use of WES and WGS. ${ }^{15}$ Recently, WGS has been used to diagnose severely-ill infants in neonatal intensive care. ${ }^{16}$ Whole-genome techniques might be applied in different settings (diagnosis in patients with symptoms, research, pharmacogenomics, presymptomatic testing and populationscreening programs) each of which raise different questions. The focus of this paper will be on the challenges presently encountered in the diagnostic field. We will also briefly highlight challenges of some of the other settings.

\section{The advantages and challenges of WGS and WGA}

An obvious advantage of next-generation sequencing techniques is the greater potential to identify the genetic component of health problems, and probably, in the near future, at a lower cost than that of the current techniques. ${ }^{17}$ The sheer mass of data generated can reveal disease-causing alleles that could not be detected otherwise. Moreover, affordable technology that generates more genomic information may aid the translation of applications to further improve health care.

In the clinical context, the challenges of handling vast amounts of information, most of which may not be directly relevant for the patient, has prompted many groups to focus or target their initial sequencing or use filters to select relevant variants after sequencing. However, if the focus is narrowed too soon or is too restrictive, potential disease-causing alleles or regions may be missed. A targeted approach could therefore hinder the diagnostic process, ${ }^{17}$ whereas in case of using filters after sequencing new variants could more easily be added to the interpretation by adjusting the filter. An example of filtering is targeting the analysis to de novo mutations in the genome of an affected child, comparing the infants' and parental genome. ${ }^{18}$ Drawbacks of untargeted approaches are that the analysis may be too time-consuming plus the fact that a larger number of unsolicited findings and unclassified variants will burden the diagnostic process and strain the informed consent procedures.

\section{Challenges at the interface of health care and research}

In a diagnostic context, it has been argued that a high sensitivity is needed to reduce the number of false negatives. ${ }^{19}$ In establishing a diagnosis, it is crucial to know whether or not a genomic variant can be interpreted as a causal variant for a specific disorder. Genes with a proven relation to disease aetiology may therefore be the focus of initial analyses. If a causal variant cannot be detected then a wider analysis may be considered. Whereas variants in genes with uncertain clinical implications would have been disregarded in the first instance, it is unclear what status they should have in the second instance. In case of an unclassified variant in a gene with proven clinical consequences, rules for reporting are available. ${ }^{20}$ However, when variants are found in genes where the function or effect on the phenotype is unknown, more caution is warranted. It is debatable whether these variants with uncertain clinical consequences should be communicated to a patient or family members and included in a person's medical file. However, for research purposes, it certainly is important to document these genes and variants, and make the information available to other researchers. This information may at a later stage lead to a clinically relevant finding after further research and subsequent clinical diagnostic confirmation. Protocols, therefore, need to be established as to whether and how whole-genome information should be documented, shared and stored and for how long. Given the pace of these new discoveries, it is paramount that accessible biobanks and databases be created with up-to-date genotype and phenotype information on variants and patients. Resources need to be secured to ensure sustainable development of these databases. ${ }^{21}$

This approach to diagnosis could result in patients automatically becoming included in scientific research activities, with the risk that their individual interests are subordinated to the research aims of their doctors. For instance, a new and not yet understood finding may help to develop knowledge and thus be highly important from a research perspective, while in the clinic it may be, for the present, an irrelevant positive finding. Not only would such an outcome be difficult to explain to the individual, but it might also lead to stress and uncertainty for parents or patients, or even to inappropriate patient management. Current ethical and legal norms require that doctors give priority to the interests of their patients so that patients are not turned into research subjects without their informed consent. It should be clear for the patient what he or she consents to, also when a diagnosis can only be established by using new methods and also as part of ongoing research. As diagnostic testing for the purposes of health care and biobank research tend to become intertwined activities, ${ }^{22}$ relevant normative frameworks including consent procedures for diagnosis, research, disclosure and storage need to be reconsidered and, if necessary, adapted to the challenges of the new situation. For instance, many research protocols state that no information about the test results will be given to individual participants because the research findings may need to be confirmed in follow-up studies. However, as WGA may lead to the identification of variants with known clinical relevance, many people argue that individual feedback should be given if the possibility of an individual health benefit is realistic. ${ }^{23,24}$

As science progresses, knowledge on validity is constantly evolving, which calls for a flexible service provision. Thus, the question emerges as to how and when a patient should be recontacted, as new information becomes available on potentially relevant genetic variants. A general duty to recontact patients cannot be maintained given the impossibility to delimit its scope. However, balancing pros and cons may require recontacting when findings have a potentially high information value, for example, therapeutic options might emerge for some disorders. New ways of communicating via websites, forums and social media may be explored to give patients or participants access to their data or to actively recontact them. Patient-interest groups could be consulted for advice regarding this issue. With this in mind, counsellors must prepare patients and their general practitioners to deal with uncertainty, and should explain the possibility that variants of unknown significance may be found before the analysis is undertaken. There is a necessity to teach and train healthcare practitioners to follow, digest and properly interpret this 'genomic tsunami'.

\section{Unsolicited findings}

The issue of incidental findings as a challenge for diagnostic testing is not new. For instance, karyotyping for mental retardation and multiple congenital anomalies may identify chromosomal abnormalities that were not initially considered. Certainly high resolution genome-wide array analysis has increased the scale of this challenge. For example, when looking for the cause of mental retardation, a 
genetic variant may be detected that causes an increased cancer risk. ${ }^{25}$ In the case of genome-wide arrays and WGA, 'unsolicited findings' seems a more appropriate term than 'incidental findings' hitherto used, as the nature of the technique is such that in principle, data will be generated that are not related to the initial diagnostic question. It can be argued that, at this particular point, the classical distinction between diagnostic testing on the one hand and screening on the other (where screening is defined as the offer of medical testing to persons without symptoms or other indications that would make such testing clinically necessary; see next section) loses much of its sharpness. ${ }^{4}$ From an ethical point of view, this observation underscores the need for a separate justification in terms of the proportionality of using whole-genome techniques in a diagnostic setting: do the advantages outweigh the disadvantages in a specific case? The importance of clarifying a severe health problem may outweigh the potential drawbacks of a genetic test that may also lead to unsolicited findings. Whether it does should be decided by the health professionals before the test is offered and the possibility of such a scenario should be discussed with the patient (or the parents) as part of pre-test counselling. As for other presymptomatic genetic testing, patients may change their opinion after having had the blood taken for the test, and should be allowed to exert their rights of autonomy.

Distinguishing between general categories of possible findings may help to facilitate consent for testing without overburdening patients with information, and may direct the professional decision-making with regard to what findings should, in principle, be retained or returned. ${ }^{19}$ This approach would also allow patients to indicate any specific information needs or preferences, including possible claims to a 'right not to know'. However, such claims do not automatically over-ride professional responsibilities in cases where the health interests of children or family members are at stake, ${ }^{26}$ as we discuss below.

To contribute to the need for guidance, clinicians should share their experiences and establish best practices with regard to counselling and informed consent procedures, as well as the handling of unsolicited findings. ${ }^{27-29}$ As these unsolicited findings could also emerge as a clinical issue a long time after testing, the question as to how this new information should be handled and communicated to the patient should also be addressed.

\section{Population screening}

In screening (as defined in the previous section), the use of genomic information calls for a different standard regarding evidence thresholds for clinical validity and utility than that used in a diagnostic setting. Screening requires high specificity in order to reduce the number of false positives. The use of filters to select regions and variants of clinical relevance may enable WGS-based targeted screening, which may not differ much from the current approaches, yet will result in many more unclassified variants. However, unsolicited findings and outcomes of unclear significance are a well-known problem also in the context of population screening. ${ }^{30}$ Nevertheless, there is an important difference in how the scope of such screening is defined. The question would no longer be which target diseases should be included in the test panel, but which should be excluded by selective analysis of WGS/WES data. The challenge will be to avoid a broader scope in the test that is not based on a rigorous evaluation of clinical utility and other screening criteria.

One area where this challenge may occur is in preconception carrier screening, allowing reproductive options in case both partners are carriers of mutations for the same autosomal recessive disorder(s).
Commercial companies already offer screening packages in which carrier status for more than a hundred of such disorders are simultaneously tested. ${ }^{31}$ For some disorders, only a limited number of variants is included in the panel. The risk here is that couples make important reproductive choices based on test results that are still insufficiently understood, and therefore, this is not recommended as good practice. Research is being conducted on developing preconceptional tests based on targeted sequencing, where knowledge on clinical validity is continually updated. ${ }^{32}$

Neonatal screening for rare diseases is another area where the introduction of WGS/WES may widen the scope of testing beyond what can be justified in terms of the current classical screening criteria. ${ }^{33}$ Some have argued that these criteria need to be modified in order to allow WGA-based testing to be used for the purposes of personalized medicine. Although this reality is not for the near future, it has been suggested that neonatal screening would be the best setting for analysing the genomes of individuals, who might then benefit from personalized prevention and treatment during the full length of their lives. ${ }^{34}$ Another problem with this approach is that it may lead to information that only becomes relevant later in life. Revealing this information may undermine the child's right to decide for himself or herself, once mature enough to do so, what to know or not to know about his or her own health prospects.

This problem of undermining the child's future autonomy rights also arises when WGS/WES is used in the context of prenatal testing (eg, as follow-up to an abnormal ultrasound) or perhaps in the future also in the context of routine prenatal screening. ${ }^{35,36}$ At present, a non-invasive targeted approach is being developed to detect Down syndrome. This test profits from the high sensitivity and specificity reached by massive parallel sequencing and a focused analysis: the numerical comparison of fetal DNA fragments in maternal blood. ${ }^{37}$ However, it is likely that the number of disorders tested for will increase in the near future. Although WGA/WEA information may be relevant to a decision by the woman or the couple about whether or not to carry the pregnancy to term, it should be acknowledged that this may also lead to the birth of children known to be at risk for severe late-onset disorders. Clearly, this outcome is at odds with ESHG recommendations concerning genetic testing of minors. ${ }^{38}$ Moreover, there may be a tension between the aim of reproductive screening (enhancing autonomy by providing meaningful reproductive options) and the fact that widening the scope of testing will make counselling and decision-making more difficult.

\section{Informational privacy and family relations}

Further exploration is needed regarding the ethical, legal and social implications of generating genomic data and information in the context of diagnostics or population screening, in view of questions related to informational privacy. For instance, should the raw data obtained through WGS be stored and if so under what conditions? It may well be that when prices drop, it will become cheaper and also more suitable from an ethical and legal point of view to perform a new WGS procedure whenever required for clinical diagnostics or screening. Potential implications for insurance and employment also need to be addressed.

Other issues that require further guidance are how to deal with information that patients or parents have indicated they would not want to receive, but that may still be important for their own health, for that of their children or any close relatives. Moreover, how to deal with outcomes that may be clinically relevant but will or may only affect the child in its adult life ${ }^{39}$ For instance, if a diagnostic test aimed at finding the cause of a hitherto unexplained disease finds that 
a girl is at risk of hereditary breast and ovarian cancer, difficult questions arise as to how to best respect her future autonomy rights without depriving her of what may be life-saving information, while also taking account of the possible health or reproductive interests of family members.

\section{Commercial applications}

The clinical utility of a test refers to the positive risk-benefit ratio and meaningful options in case of a positive result. Whereas in a growing number of countries criteria including clinical utility must be met in order for a diagnostic test or a screening programme to be responsibly introduced in health care, ${ }^{23}$ a different perspective may be taken by providers and consumers of genomic tests that can be purchased directly from companies without the intervention of a health-care professional. From the perspective of the individual's right to know about his or her genome, limiting regulations may sometimes be regarded as unnecessarily paternalistic and interfering.

Previously, the PPPC has discussed genetic testing for common complex disorders, as well as genetic testing in commercial settings. ${ }^{40,41}$ These earlier statements on the importance of clinical utility as the central criterion to assess testing and screening possibilities certainly also apply to whole-genome techniques. Whole-genome testing for common disorders would often not satisfy the criteria nowadays used to evaluate screening strategies, because of their limited clinical utility. ${ }^{42}$ Ideally, independent information about the pros and cons of WGS should be available to the public based on expert judgements from professionals explaining the issues at stake. ${ }^{43}$

\section{CONCLUSION}

Many of the issues related to WGS mentioned in this paper are not entirely new, but the scale of the challenges certainly is. For instance, the number of unsolicited findings and the amount of information becoming available substantially surpass our current experiences. As our frameworks and guidelines for offering good clinical services and sound screening are based on these experiences, the question arises as to whether these frameworks should limit new developments, or whether those developments require existing frameworks to be reconsidered and adapted. This need not mean that the technology is allowed to determine what will be offered in health care, the socalled 'technological imperative'. There is a clear need for professionals from various disciplinary backgrounds to contribute to sustainable new frameworks that allow building new health-care practices in a responsible way. $^{23}$

In order to contribute to developing best practices in implementing WGS/WES into health care, stakeholders from relevant fields in research and the clinic should set up structures for sharing experiences and establish guidelines with regard to clinical utility, counselling and informed consent procedures, as well as the handling of unsolicited findings and variants with uncertain clinical implications. Examples can be found in local, national and international initiatives, such as in EuroGentest (http://www.eurogentest.org/professionals/ web/info/public/WP8survey.xhtmland) and TECHGENE (http:// www.techgene.eu) funded by the European Union, and the Leuven University initiative in Belgium (http://www.kuleuven.be/metaforum/ docs/pdf/wg_14_e.pdf). In addition, patient and public experiences can be used to discuss and learn how to incorporate new genomic technologies in our daily lives.

Protocols can be adjusted as knowledge progresses on clinical utility and patient and public responses evolve. Any current recommendations, including the following ones, are therefore expected to be in need of re-evaluation in a few years' time.

\section{RECOMMENDATIONS}

(1) In order to contribute to developing best practices in implementing WGS/WES into health care, stakeholders from relevant fields in research and the clinic should set up structures for sharing experiences and establish testing guidelines at local, national and international levels.

(2) When in the clinical setting either targeted sequencing or analysis of genome data is possible, it is preferable to use a targeted approach first in order to avoid unsolicited findings or findings that cannot be interpreted. Filtering should limit the analysis to specific (sets of) genes. Known genetic variants with limited or no clinical utility should be filtered out (if possible neither analyzed nor reported).

(3) The use of genome-wide arrays or WGA requires a justification in terms of necessity (the need to solve a clinical problem) and proportionality (the balance of benefits and drawbacks for the patient).

(4) Whenever the use of these techniques is considered, a protocol has to be in place to give guidance on the reporting of unsolicited findings. If the detection of an unsolicited genetic variant is indicative of serious health problems (either in the person tested or his or her close relatives) that allow for treatment or prevention, in principle, a health-care professional should report such genetic variants.

(5) Guidelines for informed consent regarding diagnostic testing need to be developed. Patients' claims to a right not to know do not automatically over-ride professional responsibilities when the patient's own health or that of his or her close relatives are at stake. Patient groups could provide important input into how this should be handled.

(6) As testing for health care and for biobank research can be intertwined activities, clinicians should be aware of the importance of safeguarding the patient's position and explain the potential crossover with research. Relevant normative frameworks including consent procedures for diagnosis, research, disclosure and storage need to be reconsidered, and if necessary adapted to the challenges of the new situation.

(7) In case of testing minors, guidelines need to be established as to what unsolicited information should be disclosed in order to balance the autonomy and interests of the child and the parental rights and needs (not) to receive information that may be in the interest of their (future) family.

(8) In the case where new scientific evidence of clinical relevance to patients arises from the initial investigation after a diagnostic question was dealt with, the possibility of recontacting participants should be considered. A guideline should be established detailing how and when this should be done.

(9) To facilitate the interpretation of genome data, international collaboration is needed to build sustainable databases on genotypic and phenotypic information of variants and patients.

(10) A sustained effort at genetic education of health-care professionals is required at various levels: in primary care to inform and refer people appropriately, and in specialized care to counsel or refer patients, and to discuss and interpret genetic test results adequately.

(11) Genetic experts should engage in discussing new developments in genetics, and explain the pros and cons of genetic testing and screening in clinical and commercial settings to inform the public and raise public awareness. Enhancing genetic literacy in patients and the lay public will help to involve wider society in this debate. 


\section{CONFLICT OF INTEREST}

The authors declare no conflict of interest.

\section{ACKNOWLEDGEMENTS}

We thank all ESHG members and experts who have contributed to earlier drafts of these recommendations: Conny van Ravenswaaij-Arts, Jacqui Beckmann, Raoul Hennekam, Lidewij Henneman, Tessa van der Valk on behalf of the Dutch Genetic Alliance VSOP, Quinten Waisfisz. We further thank all participants of the PPPC meeting in Amsterdam in January 2011 that was organized in collaboration with the TECHGENE project, including Frank Baas, Anthony Bailey, Niels Nijsingh and Terry Vrijenhoek. This work has been supported by the TECHGENE project, grant agreement number 223143, funded by the EC Seventh Framework Programme theme FP7 -HEALTH2007-B. In 2011 and 2012, members or observers of PPPC were Nurten Akarsu, Pascal Borry, Anne Cambon-Thomsen, Florence Fellmann, Francesca Forzano, Shirley Hodgson, Heidi Howard, Hülya Kayserili, Christine Patch, Borut Peterlin, Dragica Radojkowic, Wolf Rogowski, Jorge Sequeiros, Maria Soller, Aad Tibben and Lisbeth Tranebjaerg; Martina Cornel was Chair of PPPC.

1 Service RF: The race for the $\$ 1000$ genome. Science 2006; 311: 1544-1546.

2 Wetterstrand KA: DNA Sequencing Costs: Data from the NHGRI Genome Sequencing Program (GSP). Available at. www.genome.gov/sequencingcosts. (accessed 10 2012).

3 Hastings R, Wert Gde, Fowler B et al: The changing landscape of genetic testing and its impact on clinical and laboratory services and research in Europe. Eur J Hum Genet 2012; 20: 911-916.

4 Dondorp W, de Wert G: Health Council of the Netherlands. The 'thousand-dollar genome': an ethical exploration. Monitoring Report Ethics and Health, 2010/2. The Hague: Centre for Ethics and Health, 2010. Publication number Health Council of the Netherlands: 2010/15E.

5 Sikkema-Raddatz B, Sijmons RH (eds): Special issue: Focus on CNV detection with diagnostic arrays. Hum Mut 2012; 33: 905-1019.

6 The 1000 Genomes Consortium. A map of human genome variation from populationscale sequencing. Nature 2010; 467: 1061-1073 http://www.1000genomes.org/ sites/1000genomes.org/files/docs/nature09534.pdf.

$7 \mathrm{Ng} \mathrm{SB}$, Buckingham KJ, Lee $\mathrm{C}$ et al: Exome sequencing identifies the cause of a mendelian disorder. Nat Genet 2010; 42: 30-35.

8 Wheeler DA, Srinivasan M, Egholm M et al: The complete genome of an individual by massively parallel DNA sequencing. Nature 2008; 452: 872-876.

9 Lunshof JE, Bobe J, Aach J et al: Personal genomes in progress: from the Human Genome Project to the Personal Genome Project. Dialogues Clin Neurosci 2010; 12 : 47-60.

10 Ashley EA, Butte AJ, Wheeler MT et al: Clinical assessment incorporating a personal genome. Lancet 2010; 375: 1525-1535.

11 Lupski JR, Reid JG, Gonzaga-Jauregui $C$ et al: Whole-genome sequencing in a patient with Charcot-Marie-Tooth neuropathy. N Engl J Med 2010; 362: 1181-1191.

12 Roach JC, Glusman G, Smit AF et al: Analysis of genetic inheritance in a family quartet by whole-genome sequencing. Science 2010; 328: 636-639.

13 Schrijver I, Galli SJ: Between hype and hope: whole-genome sequencing in clinical medicine. Personal Med 2012; 9: 243-246.

14 Gerlinger M, Rowan AJ, Horswell S et al: Intratumor heterogeneity and branched evolution revealed by multiregion sequencing. N Eng/ J Med 2012; 366: 883-892.

15 Vissers LELM, Ligt J de, Gilissen C et al: A de novo paradigm for mental retardation. Nat Genet 2010; 42: 1109-1112.

16 Saunders CJ, Miller NA, Soden SE et al: Rapid whole-genome sequencing for genetic disease diagnosis in neonatal intensive care units. Sci Trans/ Med 2012; 4: 154ra135.

17 Heger M: Dutch study aims to demonstrate cost-effectiveness of reimbursing for exome sequencing Dx. http://www.genomeweb.com/sequencing/dutch-study-aimsdemonstrate-cost-effectiveness-reimbursing-exome-sequencing-dx.

18 Veltman JA, Brunner HG: De novo mutations in human genetic disease. Nat Rev Genet 2012; 13: 565-575.
19 Berg JS, Khoury MJ, Evans JP: Deploying whole genome sequencing in clinical practice and public health: meeting the challenge one bin at a time. Genet Med 2011; 13: 499-504.

20 Bell J, Bodmer D, Sistermans E, Ramsden SC: Practice guidelines for the interpretation and reporting of unclassified variants (UVs) in clinical molecular genetics. Clinical Molecular Genetics Society 2007; http://www.cmgs.org/BPGs/pdfs\%20current\% 20bpgs/UV\% 20GUIDE.

21 deLeeuw N, Dijkhuizen T, Hehir-Kwa JY et al: Diagnostic interpretation of array data using public databases and internet sources. Hum Mutat 2012; 33: 930-940.

22 Meulenkamp TM, Gevers SK, Bovenberg JA, Koppelman GH, van Hylckama Vlieg A Smets EM: Communication of biobanks' research results: what do (potential) participants want? Am J Med Genet A 2010; 152A: 2482-2492.

23 Wright C, Burton H, Hall A et al: Next steps in the sequence. The implications of whole genome sequencing for health in the UK. PHG Foundation 2011, ISBN 978-1907198-08-3.

24 Bredenoord AL, Kroes HY, Cuppen E, Parker M, Van Delden JJM: Disclosure of individual genetic data to research participants: the debate reconsidered. Trends Genet 2011; 27: 41-47.

25 Schwarzbraun T, Obenauf AC, Langmann A et al: Predictive diagnosis of the cancer prone Li-Fraumeni syndrome by accident: new challenges through whole genome array testing. J Med Genet 2009; 46: 341-344.

26 Dondorp W, Sikkema-Raddatz B, de Die-Smulders C, de Wert G: Arrays in postnatal and prenatal diagnosis: an exploration of the ethics of consent. Hum Mutat 2012; 33 916-922.

27 Solomon BD, Hadley DW, Pineda-Alvarez DE et al: Incidental medical information in whole-exome sequencing. Pediatrics 2012; 129: e 1605.

28 Thorogood A, Knoppers BM, Dondorp WJ, De Wert GMWR: Whole genome sequencing and the physician. Clin Genet 2012; 81: 511-513.

29 Vermeesch JR, Brady PD, Sanlaville D, Kok K, Hastings RJ: Genome-wide arrays: quality criteria and platforms to be used in routine diagnostics. Hum Mutat 2012; 33 906-915.

30 Al-Shahi SR, Whiteley WN, Warlow C: Screening using whole-body magnetic resonance imaging scanning: who wants an incidentaloma? J Med Screen 2007; 14: 2-4.

31 Borry P, Henneman L, Lakeman P, Kate LP, Cornel MC, Howard HC: Preconceptional genetic carrier testing and the commercial offer directly-to-consumers. Hum Reprod 2011; 26: 972-977.

32 Bell CJ, Dinwiddie DL, Miller NA et al: Carrier testing for severe childhood recessive diseases by next-generation sequencing. Sci Trans/ Med 2011; 3: 65ra4.

33 Goldenberg AJ, Sharp RR: The ethical hazards and programmatic challenges of genomic newborn screening. JAMA 2012; 307: 461-462.

34 Collins FS: The language of life. DNA and the Revolution in Personalized Medicine. Harper: New York, 2010.

35 de Jong A, Dondorp WJ, de Die-Smulders CE, Frints SG, de Wert GM: Non-invasive prenatal testing: ethical issues explored. Eur J Hum Genet 2010; 18: 272-277.

36 Kitzman JO, Snyder MW, Ventura M et al: Noninvasive whole-genome sequencing of a human fetus. Sci Trans/ Med 2012; 4: 137 ra76.

37 Chiu RW, Akolekar R, Zheng YW et al: Non-invasive prenatal assessment of trisomy 21 by multiplexed maternal plasma DNA sequencing: large scale validity study. BMJ 2011; 342: c7401.

38 European Society of Human Genetics (ESHG). Genetic testing in asymptomatic minors:recommendations of the European Society of Human Genetics. Eur J Hum Genet 2009; 17: 720-721.

39 Hens K, Van El CG, Borry P et al: Developing a policy for paediatric biobanks: principles for good practice. Eur J Hum Genet 2013; 21: 2-7http://www.nature.com/ ejhg/journal/v21/n1/full/ejhg201299a.html.

40 Borry P: Statement of the ESHG on direct-to-consumer genetic testing for healthrelated purposes European Society of Human Genetics. Eur J Hum Genet 2010; 18: 1271-1273.

41 Van El CG, Cornel MC, on behalf of the ESHG Public and Professional Policy Committee: Genetic testing and common disorders in a public health framework. Recommendations of the European Society of Human Genetics. Eur J Hum Genet 2011; 19: 377-381.

42 Roberts NJ, Vogelstein JT, Parmigiani G, Kinzler KW, Vogelstein B, Velculescu VE: The predictive capacity of personal genome sequencing. Sci Transl Med 2012; 4: 133 ra58.

43 Health Council of the Netherlands. Screening: between hope and hype. The Hague: Health Council of the Netherlands, 2008; publication no. 2008/05E. http://www.gr.nl/ en/publications/screening-between-hope-and-hype-0. 